\title{
Repair of retrograde ascending dissection after descending stent grafting
}

Jahanzaib Idrees, MD, Amr Arafat, MD, Douglas R. Johnston, MD, Lars G. Svensson, MD, PhD, and Eric E. Roselli, MD

Objective: Retrograde dissection is now recognized as an important complication after thoracic endovascular aortic repair (TEVAR), but its treatment is poorly understood. Our objectives were to investigate the risks, describe the repair methods, and assess the outcomes of this complication.

Methods: From 2000 to 2012, 766 patients underwent TEVAR. Of these patients, 14 (1.8\%), plus 1 who had undergone TEVAR elsewhere $(\mathrm{n}=15)$, developed retrograde dissection after stent grafting. They had undergone TEVAR for distal aortic dissection in 7, intramural hematoma in 5, aneurysm in 2, and transection in 1 . Their mean age was $65 \pm 9$ years. At the initial TEVAR, the left subclavian artery was covered in 9 , the mean stent graft diameter was $34 \pm 2 \mathrm{~mm}$, and $>1$ device was used in 8 patients. The site of entry tear was at the greater curvature in 11 and lesser curvature in 4 . One patient ruptured and died 12 days after TEVAR and never made it to the operating room. The other 14 underwent proximal aortic repair. The median interval between TEVAR and repair of retrograde dissection was 6 months; 3 patients presented within 1 month. The repair techniques included reverse frozen elephant trunk in 5, total arch repair in 4, ascending or hemiarch repair in 4 , and ascending TEVAR in 1 . Concomitant procedures included aortic valve repair in 4 , replacement in 2 , root remodeling in 1 , and coronary bypass in 1 .

Results: No operative mortality occurred. One patient underwent reoperation for bleeding. Two required a tracheostomy for respiratory failure. However, no renal failure, stroke, or spinal injury occurred. At a median follow-up of 26 months, 4 aortic reoperations had occurred: 1 distal stent graft extension for type $1 \mathrm{~b}$ endoleak, 2 hybrid thoracoabdominal completion repairs for growth of residual distal disease, and 1 emergency TEVAR for aortobronchial fistula. The latter patient died of septic complications, and 3 other late noncardiac deaths occurred.

Conclusions: Retrograde ascending dissection can present as an early or a late complication after descending stent grafting because of aortic instability or disease progression and has usually been associated with descending dissection or intramural hematoma. It is a life-threatening complication that can be managed safely with early recognition and rapid delivery of open or hybrid repair. (J Thorac Cardiovasc Surg 2014;147:151-4)

The improved outcomes and lower risk associated with thoracic endovascular aortic repair (TEVAR) have led to the expansion of its use for various indications, including aortic dissection. Retrograde ascending dissection (RAD) is a feared complication after TEVAR and occurs in $1 \%$ to $3 \%$ of patients. ${ }^{1-4}$ It is an emergency that requires surgical repair.

RAD has become a well-recognized complication, and various risk factors have been suggested that are either

From the Department of Thoracic and Cardiovascular Surgery, Heart and Vascular Institute, Cleveland Clinic, Cleveland, Ohio.

Disclosures: Authors have nothing to disclose with regard to commercial support.

Read at the 39th Annual Meeting of The Western Thoracic Surgical Association, Coeur d'Alene, Idaho, June 26-29, 2013.

Received for publication June 27, 2013; revisions received Aug 6, 2013; accepted for publication Aug 21, 2013; available ahead of print Oct 21, 2013

Address for reprints: Eric E. Roselli, MD, Department of Thoracic and Cardiovascular Surgery, Cleveland Clinic, 9500 Euclid Ave, Desk J4-1, Cleveland, OH 44195-5108 (E-mail: roselle@ccf.org).

$0022-5223 / \$ 36.00$

Copyright (c) 2014 by The American Association for Thoracic Surgery

http://dx.doi.org/10.1016/j.jtcvs.2013.08.075 patient specific or device related. These have included the presence of dissection, intramural hematoma, and a connective tissue disorder. The potential risk factors attributed to the procedure include balloon angioplasty and a landing zone in the more proximal aorta. For the stent graft device, proximal bare stents and barbs have been implicated.

The management options have not been as welldescribed, with only a few studies with a small number of patients having reported this complication. We reviewed our experience with TEVAR and sought to investigate the risk factors for retrograde dissection, describe repair options, and assess outcomes.

\section{METHODS}

From 2000 to 2012, 766 patients underwent TEVAR, and $15(1.8 \%)$ presented with retrograde dissection after TEVAR performed for type B dissection $(n=7)$, intramural hematoma $(n=5)$, aneurysm $(n=2)$, and transection $(n=1)$. Fourteen patients presented acutely with chest and/or back pain at the emergency department or were sent by life flight to the Cleveland Clinic at the onset of symptoms. However, in 1 patient, the diagnosis was made subacutely by echocardiography. Thus, 14 of 15 patients underwent repair during the acute phase of dissection The mean 


\section{Abbreviations and Acronyms \\ RAD $=$ retrograde ascending dissection \\ TEVAR $=$ thoracic endovascular aortic repair}

age at presentation was $65 \pm 9$ years. At the initial TEVAR, the left subclavian artery was covered in 9 patients, and 4 patients underwent concomitant stenting of the left common carotid or innominate artery. The mean stent graft diameter was $34 \pm 2 \mathrm{~mm}$, and $>1$ device was used in 8 patients. The site of entry tear was at the greater curvature in 11 and lesser curvature in 4 (Table 2).

One patient developed a rupture and died 12 days after TEVAR, never making it to the operating room. This patient had initially undergone open repair for thoracoabdominal dissection and later developed progressive aneurysm in the more proximal thoracic descending aorta, which was stent grafted with a fenestrated device, coverage of the left subclavian artery, and concomitant stenting of the left carotid artery. The diagnosis of RAD was made at autopsy. The other 13 patients, plus 1 patient who had undergone TEVAR elsewhere, underwent proximal aortic repair. The median interval between TEVAR and repair of RAD was 6 months; 3 patients presented within 1 month and 12 presented later during follow-up (Table 2). The repair techniques included reverse frozen elephant trunk in 6, total arch repair in 4, ascending or hemiarch repair in 4, and ascending TEVAR in 1 . Concomitant procedures included aortic valve repair in 4 , aortic valve replacement in 2 , root remodeling in 1 , and coronary bypass in 1 .

\section{Follow-up}

At our institution, the patients with aortic disease were followed up with routine computed tomography imaging surveillance at discharge, within the first 3 months, at 12 months after surgery, and then annually thereafter. Of the 766 patients who had undergone underwent TEVAR, follow-up data were available from the cardiovascular information registry at Cleveland Clinic for $88 \%$, and the median follow-up period was 4 years, with follow-up data available for $\leq 8$ years for $10 \%$ of the patients.

\section{Reverse Frozen Elephant Trunk}

The reverse frozen elephant trunk is the reversed modification of the single-stage frozen elephant trunk technique and has been previously described. ${ }^{5,6}$ It was the most commonly performed operation in the present series. In brief, through a median sternotomy with hypothermic circulatory arrest (with or without selective brain perfusion), ascending and total arch replacement was performed, with separate reimplantation of the arch vessels. The distal end of the surgical graft was then sutured directly to the stent graft and native descending aorta (Figure 2). Two patients had additional stent grafts deployed antegradely at reverse frozen elephant trunk repair to extend the previous TEVAR.

\section{Ascending Hemiarch or Total Arch Replacement}

Four patients presented with RAD in which the arch seemed to be spared. However, they all had a separate entry tear found in the ascending aorta (Figure 1) on direct intraoperative inspection. The decision was made to perform an ascending and hemiarch repair only. In another 3 patients in whom the entry tear extended into the arch or branch vessels, total arch replacement using deep hypothermic circulatory arrest was performed without extending the repair directly to the previously placed thoracic stent graft device.

\section{Ascending Aortic Stent Graft}

This approach was used in 1 patient deemed too high risk for open surgery. ${ }^{7}$ The patient had previously undergone emergency open repair
TABLE 1. Potential risk factors for retrograde dissection $(n=15)$

\begin{tabular}{lr}
\hline \multicolumn{1}{c}{ Risk factor } & $\mathbf{n}(\%)$ \\
\hline Patient related & \\
Type B dissection/intramural hematoma & $12(80)$ \\
Disease progression & $7(47)$ \\
Procedure or device related & \\
Arch and/or ascending proximal landing zone & $9(60)$ \\
Zone 0 & $2(13)$ \\
Zone 1 & $2(13)$ \\
Zone 2 & $5(33)$ \\
Zone 3-4 & $6(40)$ \\
Common carotid stent & $4(27)$ \\
Proximal bare spring & $3(20)$ \\
Proximal barbs & $5(33)$ \\
\hline
\end{tabular}

of type B dissection complicated by aortoesophageal fistula that required esophagectomy and subsequent colon interposition. She had also undergone underwent hybrid thoracoabdominal repair with visceral debranching and stent grafting. Eight years later, she had presented with acute type A dissection and an entry tear in the ascending aorta.

The technique involved obtaining through and through wire access across the left ventricular apex and left subclavian artery. Under fluoroscopic guidance, a $32 \times 58$-mm aortic extension cuff was deployed into the ascending aorta using the transapical approach (Figure 2, A).

\section{Outcomes and Statistics}

Technical success was defined as the ability to complete the operation without mortality or type 1 and 3 endoleaks during fluoroscopic assessment of the excluded diseased segment. Reinterventions were defined as the need for another unplanned procedure to address complications after the initial repair. Renal failure was defined as the need for hemodialysis, and respiratory failure was defined as the need for reintubation or tracheostomy postoperatively. Follow-up was performed as scheduled outpatient visits, which included computed tomography angiography, which was analyzed using 3-dimensional reconstruction software (TeraRecon, San Mateo, Calif) to assess graft patency, device integrity, endoleaks, and aortic morphology.

Descriptive statistical analyses were used to present the variables for the present study. Continuous variables are presented as the median or mean \pm standard deviation and categorical variables as percentages. Data were obtained from the prospectively maintained Cardiovascular Information Registry, and the need for informed consent was waived by the institutional review board of the Cleveland Clinic.

\section{RESULTS}

No operative mortality occurred after repair of RAD, but 1 patient died soon after arrival at the emergency department before she could be brought to the operating room. The median length of stay was 14 days. One patient underwent reoperation for bleeding. Two patients required a tracheostomy for respiratory failure. No renal failure, stroke, or spinal injury occurred. At a median follow-up of 26 months, 4 patients required an aortic reoperation. These included a distal stent graft extension for type $1 \mathrm{~b}$ endoleak in the patient who had received the transapical ascending stent graft. Two patients required hybrid thoracoabdominal repair for growth of residual distal aortic 
TABLE 2. Procedural details

\begin{tabular}{|c|c|c|c|c|c|c|c|c|}
\hline \multirow[b]{2}{*}{ Pt. No. } & \multicolumn{7}{|c|}{ Previous TEVAR } & \multirow[b]{2}{*}{$\begin{array}{c}\text { Rescue operation } \\
\text { procedure }\end{array}$} \\
\hline & Indication & Device & Devices (n) & $\begin{array}{c}\text { LSCA } \\
\text { coverage }\end{array}$ & $\begin{array}{c}\text { Carotid or } \\
\text { innominate stenting }\end{array}$ & Entry tear & Interval (mo) & \\
\hline 1 & Type B & Gore & 1 & Yes & No & $\mathrm{LC}$ & 1.50 & RFET \\
\hline 2 & IMH & Gore & 3 & No & No & GC & 5.40 & Hemiarch \\
\hline 3 & Aneurysm & Cook & 2 & Yes & Yes & GC & 0.13 & Total arch \\
\hline 4 & Aneurysm & MedT & 1 & No & No & GC & 59.9 & RFET \\
\hline 5 & $\mathrm{IMH}$ & Gore & 2 & No & No & GC & 16.7 & Total arch \\
\hline 6 & IMH & Cook & 1 & Yes & No & GC & 2.10 & Ascending \\
\hline 7 & Rupture & Gore & 1 & Yes & No & GC & 1.67 & RFET \\
\hline 8 & Type B & Cook & 2 & No & No & $\mathrm{LC}$ & 8.97 & Total arch \\
\hline 9 & $\mathrm{IMH}$ & Gore & 1 & No & No & $\mathrm{LC}$ & 6.07 & Hemiarch \\
\hline 10 & $\mathrm{IMH}$ & Gore & 3 & Yes & Yes & GC & 0.23 & Total arch \\
\hline 11 & Type B & Gore & 3 & No & No & GC & 92.2 & Ascending TEVAR \\
\hline 12 & Type B & MedT & 1 & Yes & Yes & GC & 11.4 & Ascending \\
\hline 13 & Type B & MedT & 2 & Yes & No & GC & 5.07 & RFET \\
\hline 14 & Type B & Cook & 2 & Yes & Yes & $\mathrm{LC}$ & 0.40 & No operation \\
\hline 15 & Type B & Cook & 1 & Yes & No & GC & 50.0 & RFET \\
\hline $\begin{array}{l}\text { Total or } \\
\text { median }(n)\end{array}$ & - & - & 2 & $9(60)$ & $4(27)$ & - & 6 & - \\
\hline
\end{tabular}

Data in parentheses are percentages. Pt. No., Patient number; TEVAR, thoracic endovascular aortic repair; LSCA, left subclavian artery; Type B, type B dissection; Gore, Gore-Tex (W. L. Gore \& Associates, Newark, Del); LC, lesser curvature; RFET, reverse frozen elephant trunk; IMH, intramural hematoma; Cook, Cook, Zenith (Cook, Inc, Bloomington, Ind); $G C$, greater curvature; MedT, Medtronic (Medtronic, Inc, Minneapolis, Minn).

disease. Emergency TEVAR for an aortobronchial fistula was performed in 1 patient. The latter patient died of septic complications, and 3 other late noncardiac deaths occurred.

\section{DISCUSSION}

Emergency repair of RAD is complex and carries a high risk. It often requires ascending and arch reconstruction with hypothermic circulatory arrest. Previous studies have reported mortality of $27 \%$ to $57 \% .^{1-3}$ Our experience with open and hybrid repair of RAD after TEVAR has demonstrated the feasibility, safety, and effectiveness for management of this life-threatening complication.

Although multiple risk factors have been suggested to explain causation (Table 1), the number of events have been too small to make definitive conclusions. Nonetheless, our experience with these 15 patients can provide some important insight about this complication (Table 2). In most patients, the indications for the initial TEVAR were intramural hematoma or dissection involving the distal aorta. Although this strategy works well for acute indications, the patients' aortic tissue will be fragile, and it must be assumed that the nondissected portions of aorta could also be vulnerable. The untreated native aortic segments remain at continued risk of disease progression, and the course of RAD has demonstrated that it can present as an early or late complication after TEVAR. In our series, only 4 patients developed this complication within 30 days of TEVAR, and the interval to the rescue operation was $\geq 6$ months in 7 patients $(47 \%)$. The finding that so many of these occurred in a delayed fashion suggests that the RAD might not have been directly related to TEVAR.
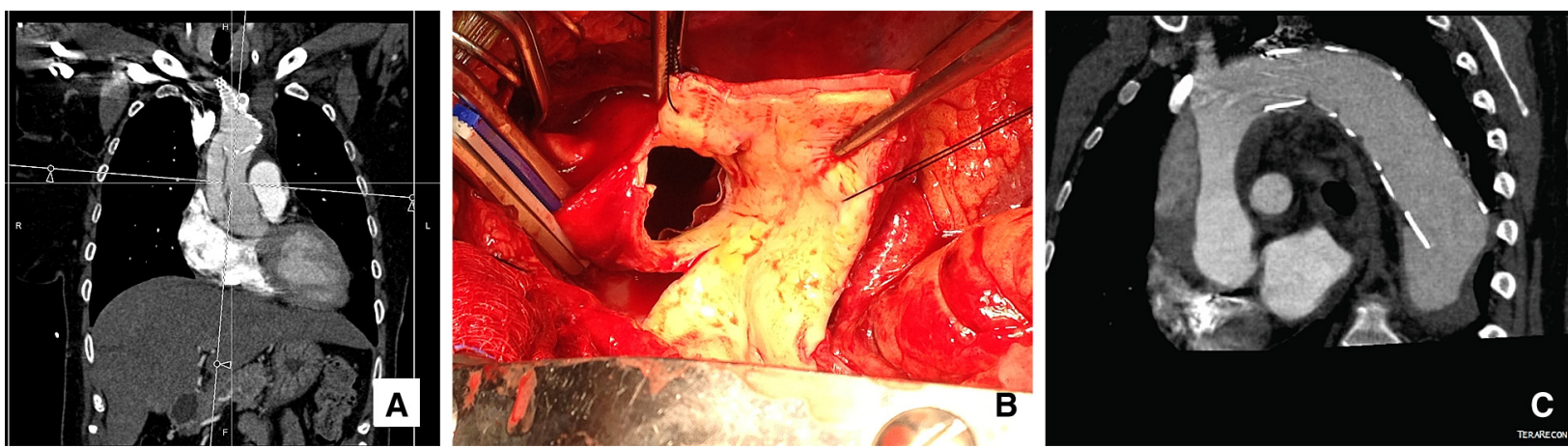

FIGURE 1. Retrograde ascending dissection. A, Computed tomography scan showing dissection tear extending from the stent graft. B, Intraoperative image showing entry tear remote from the stent graft device. C, Computed tomography scan showing entry tear in the ascending aorta. 

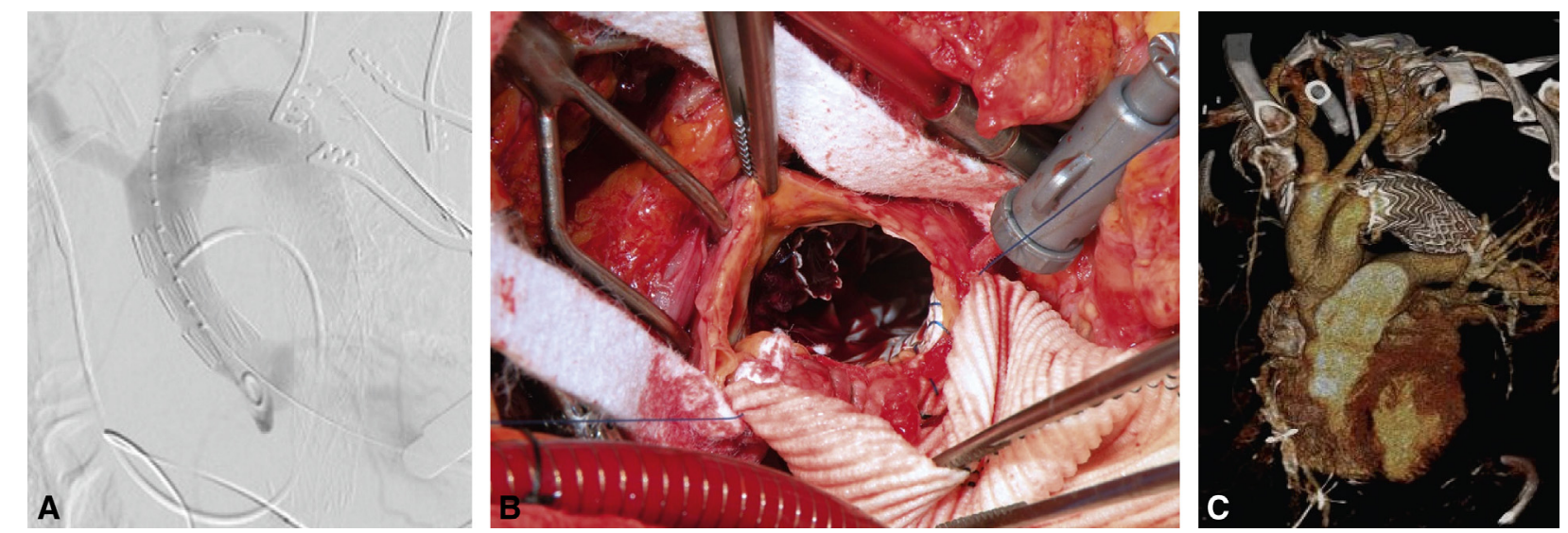

FIGURE 2. Repair methods. A, Ascending thoracic endovascular aortic repair, with fluoroscopic image showing the stent graft in the ascending aorta. B, Intraoperative image of reverse frozen elephant trunk. C, Postoperative computed tomography scan showing stable repair after reverse frozen elephant trunk.

Rather, these patients might have experienced progression of their underlying aortic disease.

Most patients in our experience had landing zones in the more proximal aorta, including several patients who underwent combined interventions on the left common carotid artery. Most, but not all, stent graft devices had barbs, and some had a proximal bare stent. One must be careful about making associations between RAD and these features of the stent graft on the basis of our experience, because the number of each brand of device used varied. At our institution, most patients undergoing TEVAR will have initially received devices with barbs as a part of large investigational device exemption trials. In both the present and other series, all the commercially available devices for thoracic stent grafting had been associated with RAD.

An important point for discussion regarding the mechanism of this complication was that most of these devices were used outside the instructions for use-by either disease indication or device position. We were limited in our ability to assess the relationship between the entry tear and the device because of the retrospective nature of the present study. Although we did not find that the entry tears were directly associated with the stent graft itself in most patients, the tears did occur that way in a few. From the computed tomography scan analysis, we found that the entry tear was along the lesser curve in 4 patients and the greater curve in the rest. The commercially available stent graft devices used in the present study lack the conformability necessary to treat very proximal arch disease that is often required in patients with dissections. Newer devices with improved conformability will hopefully reduce the incidence of this complication.

The operative strategies regarding the rescue of these patients from this complication are similar to those for acute type A dissection. It is critical to stabilize the proximal aorta; however, many patients will require extension of the repair into the arch. In our series, the choice of procedure type was determined by the intraoperative findings and surgeon preference. The reverse frozen elephant trunk procedure or total arch replacement were the preferred choices for patients with a dissection tear involving the arch, and ascending and hemiarch repair was sufficient in patients with the dissection limited to the proximal aorta.

\section{CONCLUSIONS}

RAD can present as an early or a late complication after descending stent grafting because of aortic instability or disease progression and has usually been associated with descending dissection or intramural hematoma. It is a lifethreatening complication that can be managed safely with early recognition and rapid delivery of open or hybrid repair. All patients with aortic dissection require lifelong imaging surveillance.

\section{References}

1. Eggebrecht H, Thompson M, Rousseau H, Czerny M, Lönn L, Mehta RH, et al Retrograde ascending aortic dissection during or after thoracic aortic stent graft placement: insight from the European registry on endovascular aortic repair complications. Circulation. 2009;120(11 Suppl):S276-81.

2. Dong ZH, Fu WG, Wang YQ, Guo da Q, Xu X, Ji Y, et al. Retrograde type A aortic dissection after endovascular stent graft placement for treatment of type B dissection. Circulation. 2009;119:735-41.

3. Kpodonu J, Preventza O, Ramaiah VG, Shennib H, Wheatley GH III, RodriquezLopez J, et al. Retrograde type A dissection after endovascular stenting of the descending thoracic aorta: is the risk real? Eur J Cardiothorac Surg. 2008;33: 1014-8.

4. Dumfarth J, Michel M, Schmidli J, Sodeck G, Ehrlich M, Grimm M, et al. Mechanisms of failure and outcome of secondary surgical interventions after thoracic endovascular aortic repair (TEVAR). Ann Thorac Surg. 2011;91: 1141-6.

5. Lima B, Roselli EE, Soltesz EG, Johnston DR, Pujara AC, Idrees J, et al. Modified and "reverse" frozen elephant trunk repairs for extensive disease and complications after stent grafting. Ann Thorac Surg. 2012;93:103-9.

6. Roselli EE, Sepulveda E, Pujara AC, Idrees J, Nowicki E. Distal landing zone open fenestration facilitates endovascular elephant trunk completion and false lumen thrombosis. Ann Thorac Surg. 2011;92:2078-84.

7. Roselli EE, Brozzi N, Albacker T, Lytle BW. Transapical endovascular ascending repair for inoperable acute type a dissection. JACC Cardiovasc Interv. 2013;6: $425-6$ 